\title{
An elderly-onset rheumatic syndrome: is it RS3PE?
}

\author{
Marta Oliveira, Filipa Ferreira, Erica Ferreira, Rosa Jorge \\ Internal Medicine Department, Centro Hospitalar Baixo Vouga, E.P.E. Aveiro, Portugal
}

\begin{abstract}
It is presented a case of a 75-year-old Caucasian male patient who was admitted to hospital with a history of painful swollen hands and feet, associated with worsening mobility. Examination revealed bilateral pitting edema of dorsum of hands and feet. Initial blood test showed $10.7 \mathrm{~g} / \mathrm{L}$ hemoglobin with normochromic and normocytic anemia, raised inflammatory markers (erythrocyte sedimentation rate $60 \mathrm{~mm}, \mathrm{C}$-reactive protein $16.73 \mathrm{mg} / \mathrm{dL}$ ) and normal white blood cells count. Autoantibody screen and rheumatoid factor were negative, with positive HLA-B27. Radiological findings of hands and feet did not show any erosions. The patient was also screened and investigated for associated malignancies; the tumor markers and the computed tomography thorax/abdomen were reported as normal. The patient responded extremely well to low dose prednisolone at $20 \mathrm{mg}$ daily dosage. Further follow up 5 weeks later on tapering dose of prednisolone showed complete resolution of signs and symptoms without any further flare-ups. Remitting seronegative symmetrical synovitis with pitting edema is a definite syndrome, a rare subset of polyarthritis with favorable outcome and has a good prognosis in older patients. This case illustrates the interesting diagnosis exercise that is needed, once the multiple conditions that may be related with the disease support that this is a syndrome with potentially heterogeneous etiologies. Looking for underlying malignancy is recommended.
\end{abstract}

\section{Introduction}

In 1985, McCarty and colleagues first described remitting seronegative symmetrical synovitis with pitting edema (RS3PE) as a distinct form of seronegative rheumatoid arthritis (RA)-like polyarthritis that primarily affects elderly men. ${ }^{1}$ Currently, the literature

Correspondence: Marta Sofia Oliveira, Internal Medicine Department, Centro Hospitalar Baixo Vouga, E.P.E. Aveiro, Av. Artur Ravara, 3814 Aveiro, Portugal.

Tel.: 234.378.300.

E-mail: martasoliv@gmail.com

Key words: Rheumatic; polymyalgia; synovitis; edema; paraneoplastic.

Contributions: MA, lead author; literature review and text editing; FF, literature review and text editing; EF, literature review and validation of the scientific content; RJ, literature review, validation of the scientific content, final approval of the version to be published.

Conflict of interest: the authors declare no potential conflict of interest.

Received for publication: 27 October 2016.

Revision received: 12 December 2016.

Accepted for publication: 6 January 2017.

This work is licensed under a Creative Commons Attribution NonCommercial 4.0 License (CC BY-NC 4.0).

CCopyright M. Oliveira et al., 2017

Licensee PAGEPress, Italy

Italian Journal of Medicine 2017; 11:335-339

doi:10.4081/itjm.2017.806 supports RS3PE as a distinct and separate syndrome. ${ }^{2}$ RS3PE is clinically characterized by pitting edema of both hands and feet, lack of subcutaneous nodules, negative rheumatoid factor (RF), absence of radiographic erosions, no association with HLA-DRB1 alleles, excellent response to glucocorticoids and a good prognosis. ${ }^{2,3}$ It has always been a matter of debate between rheumatologists as to whether to consider it a disease or a syndrome. The authors present a case report that is discussed based on a review of current literature. The authors also compare the clinical, laboratory and radiological features between various rheumatic diseases, such as rheumatoid arthritis, polymyalgia rheumatica and spondyloarthropathies, which are much more common than RS3PE, and may also present with distal extremity pain and swelling. ${ }^{2,3}$

\section{Case Report}

A 75-year-old male, with a past medical history of systemic hypertension, dyslipidemia and benign prostatic hyperplasia, was referred to the Orthopedics Department with major complaints of pain and stiffness of the shoulder girdle, as well as swelling and pain of all metacarpophalangeal joints (MCP), fourth and fifth right proximal interphalangeal joints and both wrists, for at least the previous five weeks. The onset was acute and the pain was moderate to severe in intensity, with no irradiation, but there was morning stiffness lasting for less than half an hour. The patient denied any history of previous joint, bone or back pain, fever, trauma, rash or pruritus. He was diagnosed with carpal tunnel syndrome, with a com- 
patible electromyography, and submitted to surgery. Nevertheless, there was no improvement and his joint pain worsened, associated with swollen hands and feet and less mobility, with poor response to nonsteroidal anti-inflammatory drugs. On examination, there was marked pitting tender edema of the hands extending up to the wrist joint, as well as in the ankles and feet (Figure 1A and B), with normal skin and nails and without splenomegaly. There was limitation of movements of the wrist and MCP joints, but no neurological deficit. The patient had normal range of motion of the shoulder, as well as a good mobility of the axial skeleton. His vitals were within the normal range and in the rest of his systemic examination (including locomotor) no abnormality was detected. Initial blood test showed normochromic and normocytic anemia (10.7 $\mathrm{g} / \mathrm{L}$ hemoglobin; medium globular volume of $85 \mathrm{fL}$ ), raised inflammatory markers (erythrocyte sedimentation (ESR) 60 $\mathrm{mm} / \mathrm{h}$, C-reactive protein (CRP) $16.73 \mathrm{mg} / \mathrm{dL}$ ), nor- mal white blood count and blood smear. Autoantibody screen and rheumatoid factor were negative, with positive HLA-B27. Both RF and anti-cyclic citrullinated peptides $(\mathrm{CCP})$ were negative. The $\mathrm{X}$-ray of the hands and feet was normal, without any evidence of erosions or fracture. The diagnosis of RS3PE was made, and the patient was given oral prednisolone $(20 \mathrm{mg} /$ day $)$. There was a good response, as pain started subsiding within a week, and all the symptoms were relieved within four weeks (Figure 1C and D). Later during the follow-up, he was screened for hidden occult malignancy; the tumor markers, the computed tomography thorax/abdomen, upper and lower digestive endoscopies were reported as normal. He has been in regular followup for the last 6 months, on tapering dose of prednisolone, without any residual disability or deformity or any further flare-ups. He will continue to be periodically monitored, including ultrasound surveillance of the prostatic hyperplasia.

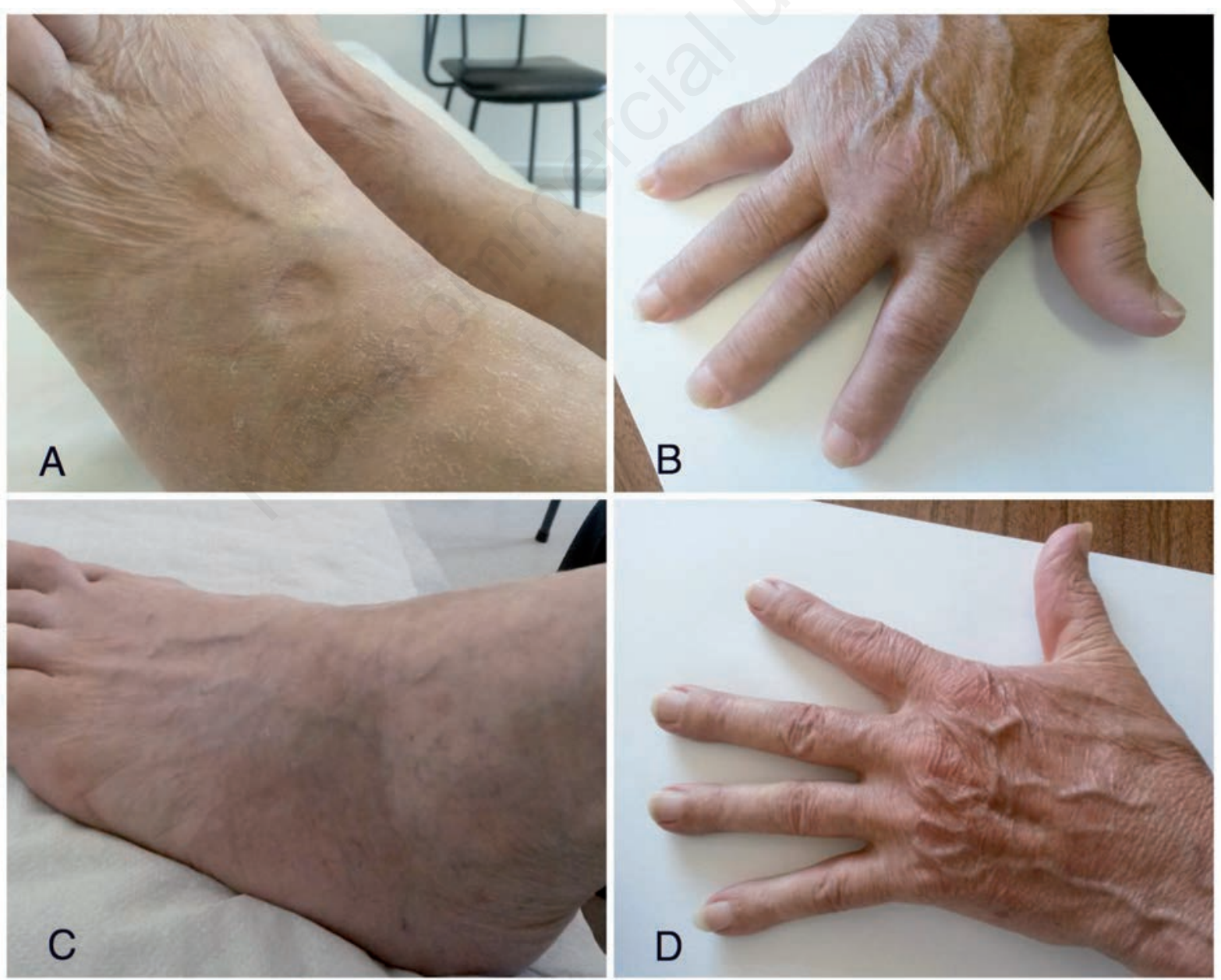

Figure 1. A) and B) Marked pitting edema of the hands, as well as in ankles and feet. C) and D) The excellent response to corticosteroid therapy, with remission of edema after 4 weeks of treatment. 


\section{Discussion}

After McCarty first described RS3PE syndrome, Olive et $_{\text {al. }}{ }^{4}$ proposed the following diagnostic criteria: bilateral pitting edema of both hands, sudden onset of polyarthritis, age more than 50 years, seronegative rheumatoid factor. The exact incidence and prevalence are not known. It affects more men than women with a ratio of $2: 1$, although a female predominance has been described in the last decade, ${ }^{5}$ and it is more frequent in the elderly. ${ }^{6}$ Since the original description, over 330 cases of RS3PE have been described. ${ }^{7}$ Most of the cases are idiopathic and fulfill the original criteria. However, many cases do not, adding new dimensions to this entity.

The laboratory parameters include elevated acute phase reactants (ESR, CRP), indicating an underlying inflammatory process. Rheumatoid factor and anti$\mathrm{CCP}$ are typically negative. Anti-nuclear antibodies are rarely positive. Varying degrees of anemia (particularly anemia of chronic diseases) may be present, which was the case of this patient. Radiologically, erosions are classically absent on X-rays. Ultrasonography/magnetic resonance imaging of the extremities have shown tenosynovitis as a major cause of subcutaneous edema. ${ }^{2,3,6,7}$ Our patient presented with all these features. He was first diagnosed with carpal tunnel syndrome, and the surgical intervention did not improve the situation. Although the electromyography results were consistent with this diagnosis, there may have been an overlap of clinical entities, hindering the diagnosis of RS3PE.

The immunopathogenesis of the disease is still in the dark. Various studies have established the role of the vascular endothelium derived growth factor (VEGF) as a major contributor to polysynovitis and subcutaneous edema of the extremities, by increasing vascular permeability. ${ }^{3}$ VEGF has potent vasodilatory effects and increases vascular permeability. ${ }^{2}$ Arima et al. measured serum VEGF levels of three RS3PE patients and compared them with those of patients with several classic connective tissue diseases (2005) and found several-fold increase of VEGF levels in patients with RS3PE over the controls. ${ }^{8}$ The elevated VEGF levels in patients with RS3PE decreased after glucocorticoid treatment, indicating a possible role of VEGF in the disease.

A central question is whether RS3PE is a distinct clinical entity or just a variant of polymyalgia rheumatica (PMR). Previous reports suggest that RS3PE is a syndrome that may represent the late beginnings of other rheumatic diseases, such as RA, spondyloarthropathies, and SS, among others. ${ }^{2,3,6,9,10} \mathrm{~A}$ study from Kimura et al. in 2012 revealed that all studied patients with RS3PE met PMR criteria, an unexpected finding. ${ }^{10}$
PMR is characterized by inflammatory pain and stiffness of the shoulder and pelvic girdles, with biochemical evidence of inflammation. It occurs most frequently in patients over 50 years of age, and is the most common rheumatic disease in older individuals. However, clinical diagnosis of PMR is rather difficult due to the large differential diagnosis that can mimic PMR, and due to the wide variation in clinical picture. ${ }^{11}$ Presenting symptoms, including proximal pain and stiffness, a commonly accepted characteristic of PMR, are not indicative of PMR and can occur in other rheumatologic diseases. ${ }^{12}$ The presented patient had no decrease in muscle strength of the scapular or pelvic girdle.

Although RS3PE and PMR share several features, they appear to be clinically distinctive. The relatively acute onset of symmetrical inflammation of joints and carpal tunnel syndrome are overlapping features. ${ }^{10}$ However, RS3PE syndrome is more common in men, involving the wrist, carpus, and flexor digitorum tendons disproportionately, and is associated with marked pitting edema of the dorsum of the hand. In contrast, PMR is more common in women, predominantly involving the shoulders and hips. The duration of PMR may be considerably longer, often many years, and flares accompanying reduction of prednisone dosage are very common. ${ }^{10,12} \mathrm{On}$ the other hand, in RS3PE most patients respond well and begin to notice significant symptomatic improvement within 24 to $72 \mathrm{~h}$ following starting glucocorticoids. Prednisone or other glucocorticoid equivalents of 10-15 mg daily are used for 2 to 3 weeks and may be tapered every week thereafter to maintain control of the disease with the lowest dosage possible for 12 to 18 months. In general, all cases treated remained well without treatment after remission. ${ }^{2,3,6,9,10,12}$ This patient noted an improvement after one week of treatment, and he was asymptomatic by the end of the fourth week. Disease-modifying antirheumatic drugs were rarely tried or needed in this disease. ${ }^{2}$ No reports of biologic use to treat RS3PE have been seen to our knowledge. Generally, patients with RS3PE without concomitant neoplasm carry a good prognosis.

The differential features of RS3PE syndrome, PMR and RA are compared in Table 1.,6,13

Facing the symptoms of this patient it was important to rule out other rheumatologic diseases. Although he had a positive HLA-B27 and elevated acute phase reactants, he did not have chronic inflammatory back pain or any signs of sacroiliitis, psoriasis, enthesitis or dactylitis. Besides, all the spinal mobility measurements were within normal ranges, which make the diagnosis of ankylosing spondylitis or psoriatic arthritis less probable.

Both cancer and benign tumors have been reported in association with RS3PE since 1985, and they in- 
Table 1. The differential features of RS3PE syndrome, polymyalgia rheumatica and rheumatoid arthritis.

\begin{tabular}{llll}
\hline & RS3PE & PMR & RA \\
\hline Onset & Sudden & May be sudden & Gradual \\
\hline Age of onset & After sixth decade & After sixth decade & Third to fifth decade \\
\hline Sex predominance & Male 2:1 & Female 2:1 & Female 2:1 \\
\hline Synovitis & Moderate & Absent & Severe \\
\hline Pitting edema & Common & Rare & Rare \\
\hline Joints & Wrist and feet & Shoulder and pelvic girdle & Hands, feet, wrist, knees \\
\hline Rheumatoid factor & Negative & Negative & Positive \\
\hline Sedimentation rate & High & Very high & Normal-high \\
\hline Response to low-dose steroids & Very good & Good & Moderate \\
\hline Remission & $6-15$ months & 24 months & No remission \\
\hline Long-term prognosis & Excellent & Frequent relapses and recurrences & Variable
\end{tabular}

RS3PE, remitting seronegative symmetrical synovitis with pitting edema; PMR, polymyalgia rheumatica; RA, rheumatoid arthritis.

clude both hematological malignancies (Hodgkin's lymphoma, leukemia) and solid tumors (prostate, gastrointestinal, lung, breast, ovary, bladder, endometrium and fibrous histiocytoma). ${ }^{14}$ In addition to the single case reports, there were several case series reports to address the incidence of malignancy in RS3PE patients. Based upon the pooled data of the above separate studies, the malignancy rate in association with European/American RS3PE patients was estimated to be $31 \%$. $^{2}$ Even though most often RS3PE presents before the discovery of a malignancy, it can occasionally present after the diagnosis of a malignancy. Overall, the current consensus is that RS3PE represents a form of paraneoplastic rheumatic syndrome..$^{2,10,12,14}$ Musculoskeletal symptoms arise in the joints, fasciae, muscles, vessels, or bones generally no longer than 2 years before the diagnosis of an associated neoplasm. ${ }^{2}$

In the neoplastic microenvironment, angiogenesis is an underlying promoter for tumor growth, invasion, and metastasis. As previously stated, VEGF plays an important role in the angiogenic process. ${ }^{15}$ It has potent vasodilatory effects and increases vascular permeability. Moreover, elevated levels of interleukin- 6 and matrix metalloproteinase-3 were reported in patients with cancer and RS3PE compared to those without neoplastic disease. ${ }^{2}$ Given that RS3PE is a heterogeneous syndrome and associated with malignancies in a significant number of cases, further study of other potential mechanisms in RS3PE patients, particularly those with neoplasia, is needed. Patients with coexisting RS3PE and malignancy tend to respond poorly to glucocorticoid therapy and to have more dramatic systemic symptoms. Treatment of the underlying malignancy in RS3PE may be important. The prognosis of these patients may depend on the underlying malignancies. $^{2}$

\section{Conclusions}

Remitting seronegative symmetrical synovitis with pitting edema is a syndrome characterized by acute onset of polyarthritis with negative rheumatoid factor, absence of joint erosions on radiographs and an exquisite response to low-dose steroids, with a sustained long-term response. An early prompt diagnosis is required, as proper treatment results on a dramatic relief of the patient, while misdiagnosis results in a more intensive and expensive therapy, over a long period of time. It may present as a paraneoplastic manifestation, especially in older people who show a poor response to steroids. Currently, there are no data to support cost-effectiveness of screening for a malignancy in the setting of RS3PE; however, ageappropriate screening is favored, particularly for non-steroid responders.

\section{References}

1. McCarty DJ, O’Duffy JD, Pearson L, Hunter JB. Remitting seronegative symmetrical synovitis with pitting edema: RS3PE syndrome. JAMA 1985;254:2763-7.

2. Li H, Altman RD, Yao Q. RS3PE: Clinical and research development. Curr Rheumatol Rep 2015;17:49.

3. Varshney AN, Singh NK. Syndrome of remitting seronegative symmetrical synovitis with pitting edema: A case series. J Postgrad Med 2015;61:38-41.

4. Olive A, del Blanco J, Pons M, et al. The clinical spectrum of remitting seronegative symmetrical synovitis with pitting edema. The Catalan Group for the Study of RS3PE. J Rheumatology 1997;24:333-6.

5. Oide T, Ohara S, Oguchi K, et al. Remitting seronegative symmetrical synovitis with pitting edema (RS3PE) syndrome in Nagano, Japan: clinical, radiological, and 
cytokine studies of 13 patients. Clin Exp Rheumatol 2004;22:91-8.

6. Salam A, Henry R, Sheeren T. Acute onset polyarthritis in older people: Is it RS3PE syndrome? Cases J 2008;1:132.

7. Karmacharya P, Donato A, Aryal M, et al. RS3PE revisited: a systematic review and meta-analysis of 331 cases. Clin Exp Rheumatol 2016;34:404-15.

8. Arima K, Origuchi T, Tamai M, et al. RS3PE syndrome presenting as vascular endothelial growth factor associated disorder. Ann Rheum Dis 2005;64:1653-5.

9. Yao Q, Su X, Altman RD. Is remitting seronegative symmetrical synovitis with pitting edema (RS3PE) a subset of rheumatoid arthritis? Semin Arthritis Rheum 2010;40:89-94.

10. Kimura M, Tokuda Y, Oshiawa H, et al. Clinical characteristics of patients with remitting seronegative symmetrical synovitis with pitting edema compared to patients with pure polymyalgia rheumatica. J Rheumatol 2012;39;148-53.
11. Muller S, Hider S, Belcher J, et al. Is câncer associated with polymialgia rheumatica? A cohort study in the General Practice Research Database. Ann Rheum Dis 2014;73:1769-73.

12. Emamifar A, Hess S, Gildberg-Mortensen R, Hansen I. Association of remitting seronegative symmetrical synovitis with pitting edema, polymyalgia rheumatica, and adenocarcinoma of the prostate. Am J Case Rep 2016;17:60-4.

13. Silva F, Méndez V. Síndrome RS3PE: remitting seronegative symmetrical synovitis with pitting edema. A presentation of 3 cases. Semergen 2012;38:472-5

14. Paira S, Graf C, Roverano S, Rossini J. Remitting seronegative symmetrical synovitis with pitting oedema: a study of 12 cases. Clin Rheumatol 2002;21:146-9.

15. Mittal K, Ebos J, Rini B. Angiogenesis and the tumor microenvironment: vascular endothelial growth factor and beyond. Semin Oncol 2014;41:235-51. 